\title{
Synthesis of Novel Heterocycles Containing Perfluoroalkyl Groups: Reaction of Perfluoro-2-methyl-2-pentene with 1,3-Binucleophilic Reagents
}

\author{
Ki-Whan Chi, ${ }^{*}$ Hyun-A Kim, Wonjon Lee, Tae-Ho Park, ${ }^{\dagger *}$ Uk Lee, ${ }^{+}$and Georgii G. Furin ${ }^{\$}$ \\ Department of Chemishy, University of Ulsan. Ulsan 680-719, Korea \\ KKorea Research Institule of Chemical Technology. Daejeon 305-606. Korea \\ ${ }^{\ddagger}$ Department of Chemistr. Pukyong National University, Busan 608-737, Korea \\ $\$$ Institule of Organic Chemistry, Nowosibirsk 630090. Russia \\ Recened March 7. 2002
}

Keywords : Perfluoro-2-methyl-2-pentene, 1,3-Binucleophilic reagent, Heterocycle. Perfluoroalkyl group. Synthesis.

It has been well known that some of lluorine-substituted organic compounds are more biologically active than the analogous organic molccules. In this sense, fluorine-containing heterocycles have recently drawn much attention because of their enhanced biological activities. Many of them are used as new medicines or pesticides as themselves or preeursors for the synthesis of biologically active compounds.? In spite of the usefulness of those compounds, there have been few facile methods for the synthesis of fluorine-containing heterocycles." Therefore, formation of heterocycles with reactive intemal perfluoroolefins ${ }^{4}$ and binucleophilic reagents must be an altractive method for the synthesis of Jluorine-containing fout- to seven- membered rings. This methodology includes nuclcophilic addition or substitution of a binucleophilic reagent to a perfluoroolelin followed by an intramolecular nucleophilic cyclization to allord a lluoro-organic heterocycle. We have systematically studied the new synthetic methodology with perfluoro-2-methyl-2-pentene (1) and various binucleophilic reagents, and found out that live-, six-or seven-membered heterocycles containing perfluoroalkyl groups could be respectively prepared by using 1,2-, 1,3- or 1,4-binucleophilic reagents. ${ }^{5}$

We have also tried to expand the applicability of this methodology, and herein we report an interesting result in the synthesis of novel heterocycles by using $\mathbf{I}$ and various 1,3 (S,N, N,N and N,C)-binucleophilic reagents. Reactions of 1 in the presence of triethylamine were scrutinized with 2mercaptobenzimidazole (2a), 2-mercapto-5-nitrobenzimidazole (2b), 2-mercaptopyrimidine (2c), 2-hydroxybenzimidazole (2d). 2-hydroxybenzothiazole (2e), 2-aminobenzothiazole (2f), 2-aminothiazole (2g), 2-amino-5-chlorobenzoxazole (2h). 1-aminonaphthalene (2i) or 2-aminonaphthalene (2j) as a 1,3-binucleophilic reagent.

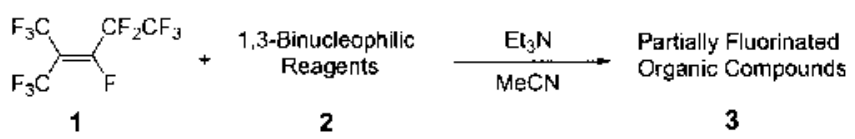

As shown in Table I, the five-membered heterocycles 3a-b were successfully synthesized by reactions between $\mathbf{l}$ and the 1,3(S,N)-binucleophilic reagents $2 \mathbf{a}-\mathbf{b}$. For example, the
Table I. The structures and isolated yields of the products 3

\begin{tabular}{|c|c|c|c|c|c|}
\hline $\begin{array}{c}\text { Nucleo- } \\
\text { phile } 2\end{array}$ & Product 3 & $\begin{array}{l}\text { Isolated } \\
\text { yield } \\
(\%)\end{array}$ & $\begin{array}{l}\text { Nucleo- } \\
\text { phile } 2\end{array}$ & Product 3 & $\begin{array}{c}\text { Isolated } \\
\text { yield } \\
(\%)\end{array}$ \\
\hline $\mathbf{2 a}$ & & 40 & $2 f$ & & 62 \\
\hline & $3 a$ & & & $\mathbf{3 f}$ & \\
\hline $2 b$ & & 60 & $2 \mathrm{~g}$ & & 12 \\
\hline & $3 b$ & & & $3 g$ & \\
\hline $2 c$ & & 59 & $2 \mathrm{~h}$ & & 47 \\
\hline
\end{tabular}

$3 c$

$3 \mathrm{~h}$ 2d<smiles>FC(F)(F)C(Oc1nc2ccccc2[nH]1)=C(C(F)(F)F)C(F)(F)F</smiles>

3d

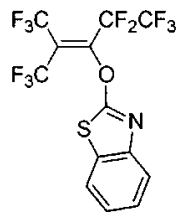

3e

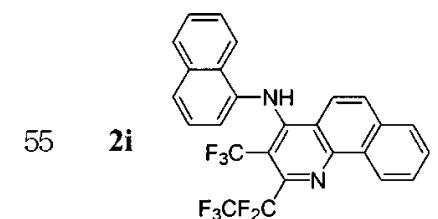

$3 i$

76

$\mathbf{2 j}$

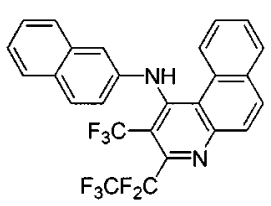

$3 \mathbf{j}$ 1,3-binucleophilic reagent $\mathbf{2 a}$ atlacks $\mathbf{1}$ in the presence of triethylamine followed by intramolecular cyclization between the regenerated electrophilic center of unsalurated intermediate 
and the second nucleophilic center in the bidentate 2a to give a five-membered ring intenmediate. Elimination of a fluoride anion from the intermediate provides the final product $3 \mathbf{a}$ with an exocyclic carbon-carbon double bond. In these reactions. triethylamine is believed to function as a nucleophilic cataly st as well as an acid scavenger. ${ }^{313}$

lt is especially noteworthy that treatment of $\mathbf{2} \mathbf{a} \mathbf{l}-\mathbf{b}$ with $\mathbf{1}$ produced the five-membered rings $\mathbf{3 a - b}$ instead of sixmembered rings. It may be attributed to the fact that formation of a fixe-membered ring is thermodymamically more favored than that of a six-membered ring with 1 and 2a-b.
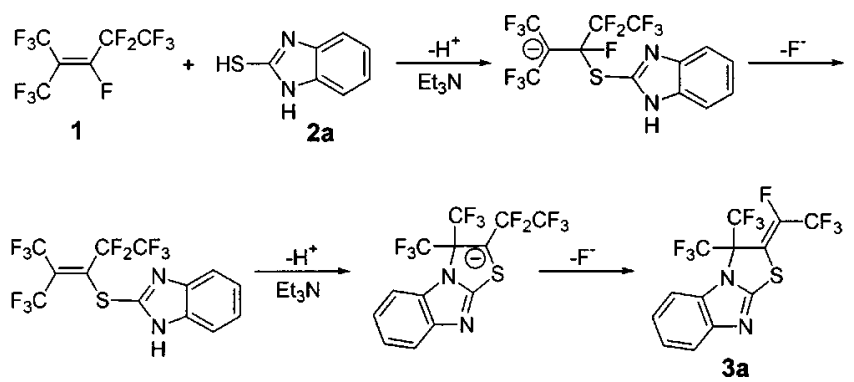

Reactions of 1 with the $1.3(\mathrm{~S} . \mathrm{N}$ or $\mathrm{O} . \mathrm{N})$-binucleophilic reagents $2 \mathrm{c}$-e, on the other hand. provided only the substituted products 3c-e. which indicates that ring formations from 3c-e are themodynamically disfavored.

The various six-membered heterocycles $\mathbf{3 f}-\mathbf{j}$ were also prepared by reactions between 1 and the $1,3($ N.N or N.C)binucleophilic reagents $\mathbf{2 f - j}$. The $1.3(\mathrm{~N} . \mathrm{N})$-binucleophilic reagent $2 f$. for instance, attacks 1 followed by intramolecular cyclization between the newly formed electrophilic center of unsaturated intermediate and the second nucleophilic center in the bidentate $2 \mathbf{f}$ to give a six-membered ring intermediate. Then aqueous work-up with the crude products provides the final product $3 \mathrm{f}^{\text {il' }}$ It is interesting to note that the carbon atom next to the amino group in the $1.3(\mathrm{~N} . \mathrm{C})$-binucleophilic reagents $\mathbf{2} \mathbf{i}-\mathbf{j}$ acts as the second mucleophilic center to form a heterocycle. Two equivalents of aminonaphthalene ( $2 \mathbf{i}$ or $\mathbf{2 j}$ ) react with one equivalent of $\mathbf{1}$ to yield the final product $3 \mathbf{i}$ or 3j. ${ }^{i c}$

In summary various five- or six-membered heterocycles 3a-b and 3f-j containing perfluoroalkyl groups were easily and effectively synthesized by reactions between 1 and the 1.3-bimucleophilic reagents $\mathbf{2} \mathbf{a}-\mathbf{b}$ and $\mathbf{2} \mathbf{f}-\mathbf{j}$ in the presence of triethylamine. The novel heterocyclic products $\mathbf{3} \mathbf{a}-\mathbf{b}$ and $\mathbf{3} \mathbf{f}-\mathbf{j}$ are expected to be biologically active compounds as themselves or useful precursors in subsequent reactions.

\section{Experimental Section}

Starting materials were purchased from the Aldrich chemical company and used without further purification. All chromatography solvents were of analytical grade and freshly distilled prior to use. Thin-layer chromatographic analy ses were conducted by using a pre-coated TLC plate $\left(60 \mathrm{~F}_{-5.7} .20 \mathrm{~cm} \times 20\right.$ $\mathrm{cm})$ purchased from the Merck company. Silica gel (230-
400 mesli) was used in flash chromatography: IR spectra were recorded on a Mattson 5000 (UNICAM) spectrometer (KBr). Melting points of the prepared compounds were detemined on an Aldricl melt temp 11 apparatus and were uncorrected. ${ }^{\mathrm{H}} \mathrm{H}$ and ${ }^{19} \mathrm{~F}$ NMR (300 MHz. $282 \mathrm{MHz}$. respectively) spectra were recorded using a Varian Unity-plus 300 FT NMR or Bruker AM-300 NMR spectrometer, and $\mathrm{CFCl}_{3}$ was used as an internal standard for ${ }^{19} \mathrm{~F}$ NMR. Mass spectra were obtained using a KRATOS Profile HV-3 or Shimadzu GC MS-Q.P 5050 (70 eV) spectrometer with a direct insertion probe. Stoe Stad-4 diffractometer was used for the crystal structure analy sis by X-ray.

General synthetic procedu re: To a solution of perfluoro2-methyl-2-pentene (1.00 g. $3.33 \mathrm{mmol}$ ) and triethylamine ( $1.01 \mathrm{~g} .10 .0 \mathrm{mmol}$ ) in acetonitrile $(30 \mathrm{~mL})$ was added at 0 ${ }^{\circ} \mathrm{C}$ a 1.3 -binucleophilic reagent $(3.33 \mathrm{mmol}){ }^{6}$ The resulting solution was stirred at $0^{\circ} \mathrm{C}$ for $1 \mathrm{~h}$, at $25^{\circ} \mathrm{C}$ for $1 \mathrm{~h}$. and 40 ${ }^{\circ} \mathrm{C}$ for $2 \mathrm{~h}$. consecutively. To the reaction mixture was added water $(20 \mathrm{~mL})$ and acetonitrile was evaporated, and organic components were extracted with methy lene chloride $(3 \times 30$ $\mathrm{mL}$ ). The combined organic extracts were washed with water $(30 \mathrm{~mL})$, brine $(30 \mathrm{~mL})$ and dried over $\mathrm{Na}_{2} \mathrm{SO}_{4}$. The organic solvents were removed under vacuo and the final products were purified by flash chromatography: The isolated products were identified by NMR, IR. MS and X-ray cry stallography ${ }^{-}$ analyses.

2-Tetrafluoroethylidene-3,3-bist rifluoromethỵl-2,3-dihydrobenzo[4,5]imidazo[2,1-b]thiazole (3a). white solid: m.p. 86-88 ${ }^{\circ} \mathrm{C}(\mathrm{MeOH})$ : ' $\mathrm{H}-\mathrm{NMR}\left(\mathrm{CDCl}_{3} 300 \mathrm{MHz}\right) \delta 7.24-$ $7.70(\mathrm{ml},+\mathrm{H}) ;{ }^{19} \mathrm{~F}-\mathrm{NMR}\left(\mathrm{CDCl}_{3} .282 \mathrm{MHz}\right) \delta-107.73-$ 107.31 (m. IF), $-68.56(\mathrm{~d} . J=2.0 \mathrm{~Hz} .3 \mathrm{~F}),-68.48(\mathrm{~d} . J=4.2$ Hz. 3F). $-68.46($ d. $J=1.7 \mathrm{~Hz}, 3 \mathrm{~F})$; IR $\left(\mathrm{cm}^{-1}\right) 3058,1525$. 1346, 1149. 963, 731, 678: MS $m z$ (rel. intensity) $+10\left(\mathrm{M}^{\prime}\right.$. 99), $341(96)$.

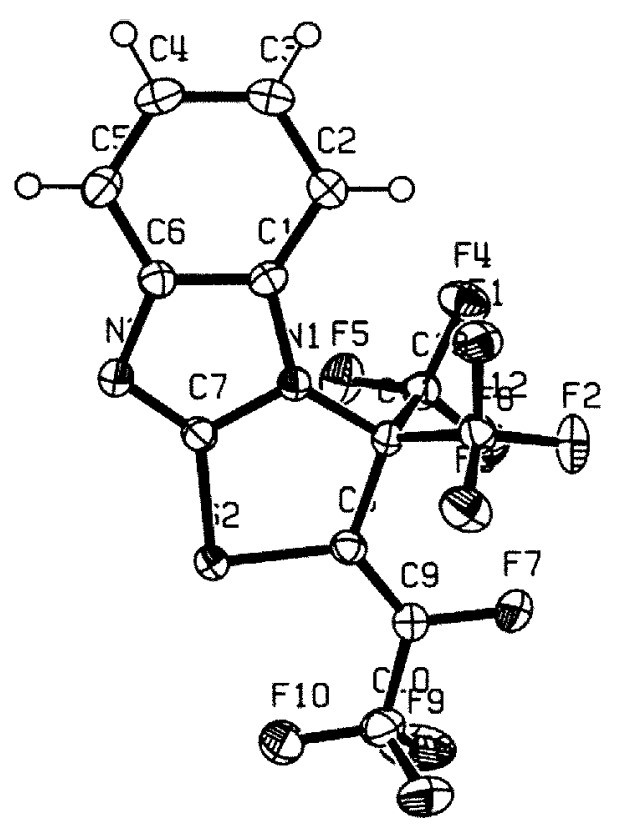

Figure 1. ORTFP drawing of $\mathbf{3 a}$. 
7-Nitro-2-tetrafluoroethylidene-3,3-bistrifluoromethyl2,3-dihydrobenzo|4,5|imidazo $[2,1-b \mid$ thiazolc (3b). whitc solid; m.p. 90-93 ${ }^{\circ} \mathrm{C}$; ${ }^{1}$ I I-NMR (CDCl $300 \mathrm{MI}$ lz) $\delta 7.27$ (s. 1H $), 7.58(\mathrm{~d}, J-8.8 \mathrm{ll} z, 1 \mathrm{Jl}), 7.79(\mathrm{~d}, J-8.8 \mathrm{Jz}, 1 \mathrm{Il}) ;{ }^{19} \mathrm{~F}-$ NMR $\left(\mathrm{CDCl}_{3,} 282 \mathrm{Mrl} z\right) \delta-105.90 \sim-105.41$ (m, IF), -68.42 $(\mathrm{d}, J-3.9 \mathrm{l} z, 3 \mathrm{~F}),-68.33(\mathrm{~d}, J-5.4 \mathrm{l}[\mathrm{z}, 3 \mathrm{~F}),-68.27$ (d, $J-$ $\left.5.4 \mathrm{HL}_{\mathrm{z}}, 3 \mathrm{~F}\right)$; IR $\left(\mathrm{cm}^{-1}\right) 3076,1599,1520,1365,1098$; MS $\mathrm{m} / \mathrm{s}$ (rel. intensity) $435\left(\mathrm{M}^{-}, 47\right), 386(80)$.

2-(3,3,3-Trifluoro-1-pentafluoroethyl-2-trifluoromethylpropenylsu Ifanyl)pyrimidine (3c). yellow liquid; ' $\mathrm{I}$-NMR $\left(\mathrm{CDCl}_{3,300 \mathrm{MH} z)} \delta 7.14(\mathrm{~L}, J-4.9 \mathrm{Jl} z, 1 \mathrm{H}), 8.57(\mathrm{~d}, J-4.9\right.$ $\mathrm{HI}, 2 \mathrm{HI}) ;{ }^{19} \mathrm{~F}-\mathrm{NMR}\left(\mathrm{CDCl}_{\mathrm{i}}, 282 \mathrm{MH} \mathrm{z}\right)-103.95$ (q. $J-20.7$ $\mathrm{H} z, 2 \mathrm{~F}),-78.20(\mathrm{q}, J-10.3 \mathrm{Jl} z, 3 \mathrm{~F}),-60.28$ (q, $J-\mathrm{I} 1.4 \mathrm{H} \mathrm{L}$, $3 \mathrm{~F}),-58.30 \sim-58.09(\mathrm{~m}, 3 \mathrm{~F}) ; \mathrm{IR}\left(\mathrm{cm}^{-1}\right) 2895,2839, \mathrm{l} 627$, $1479,1123,1031,786$; MS $m / z$ (rel. intensity) $392\left(\mathrm{M}^{+}, 7\right)$. $373(62), 323(85)$.

2-(3,3,3-Trifluoro-1-pentafluorocthyl-2-trifluoromethylpropenyloxy)-1 $\boldsymbol{H}$-benzoimidazole (3d). yellow solid; m.p. 57-58 "C.; ll I-NMR (C.DCl, $300 \mathrm{MH}$ [z) $87.00-7.06(\mathrm{~m}, 2 \mathrm{l}$ ), $7.28(\mathrm{dd}, J-3.6,5.8 \mathrm{~Hz}, 2 \mathrm{~J}) \div{ }^{19} \mathrm{~F}-\mathrm{NMR}\left(\mathrm{CDCl}_{i}, 282 \mathrm{MI} z\right)$ $\delta-111.33 \sim-110.74(\mathrm{~m}, 2 \mathrm{~F}),-80.94(\mathrm{q}, J-9.7 \mathrm{l}[\mathrm{L}, 3 \mathrm{~F}),-62.35$ (q, $J-10.4$ ll $2.3 \mathrm{~F}),-57.74 \cdots-57.43(\mathrm{~m}, 3 \mathrm{~F})$; lR $\left(\mathrm{cm}^{-1}\right) 3040$, 1762, 1487, 1201, 1033; MS $m / z$ (rel. intensity) $419\left(\mathrm{M}^{+}\right.$. $80), 373(16), 350(23), 304$ (30).

2-(3,3,3-Trifluoro-1-pentafluoroethyl-2-trifluoromethylpropenyloxy)benzothiazole (3e). yellow solid: m.p. 4850

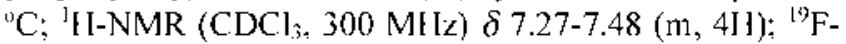
NMR (C.DCl $\left.]_{3}, 282 \mathrm{Mr} z\right) \delta-110.06 \sim 109.78(\mathrm{~m}, 2 \mathrm{~F}),-80.28$ (q. $J-9.5$ [ [z, 3F), $-62.03(\mathrm{q}, J-10.6 \mathrm{~J} / z, 3 \mathrm{~F}),-57.66 \cdots-57.38$ (m, 3F); IR $\left(\mathrm{cm}^{-1}\right)$ 3095, 1698, 1474, I193; MS $\mathrm{m} / \mathrm{z}$ (rel. intensity) $431\left(\mathrm{M}^{-}, 76\right), 362(99)$.

2-Pentafluorocthyl-3-trifluoromethyl-9-thia-1,4a-diazafluoren-4-one (3f). bright ycllow solid; m.p. $149-150^{\circ} \mathrm{C}$ (recrystallized fiom EtOAchexane); ${ }^{1} \mathrm{~J}-\mathrm{NMR}\left(\mathrm{CDCl}_{3}, 300\right.$ Ml lz) $\delta 7.65-7.70(\mathrm{~m}, 2$ I), $7.83-7.86(\mathrm{~m}$, IH [). $9.11-9.15(\mathrm{~m}$, 1H $) ;{ }^{19} \mathrm{~F}-\mathrm{NMR}(\mathrm{CDCl}, 282 \mathrm{MH} z) \delta-109.89(\mathrm{q}, j-21.9 \mathrm{Il} z$, 2F), $-80.21(\mathrm{~s}, 3 \mathrm{~F}),-58.09\left(\mathrm{t}, J-21.9 \mathrm{H}[\mathrm{z}, 3 \mathrm{~F}) ;\right.$ IR $\left(\mathrm{cm}^{-1}\right)$ $3123,17 \mid 4,1522,1338,1190 ;$ MS $m /=$ (rel. intensity) 388 $\left(\mathrm{M}^{+}, 96\right), 291$ (100), $191(53), 134$ (86).

7-Pentafluorocthyl-6-trifluoromethylthiazolo[3,2-a|pyrimidin-5-one (3g). brown solid; m.p. 76-77" C. (EtOAc/ hexanc): '] $\mathrm{l}-\mathrm{NMR}\left(\mathrm{CDCl}_{i}, 300 \mathrm{MJ} z\right)$ ) $\delta 7.36(\mathrm{~d}, J-4.8 \mathrm{[z}$. IH ), 8.20 (d, $J-4.8 \mathrm{Jl} z$, IJ $) ;{ }^{19} \mathrm{~F}-\mathrm{NMR}\left(\mathrm{CDCl}_{3,}, 282 \mathrm{MI} / z\right)$,

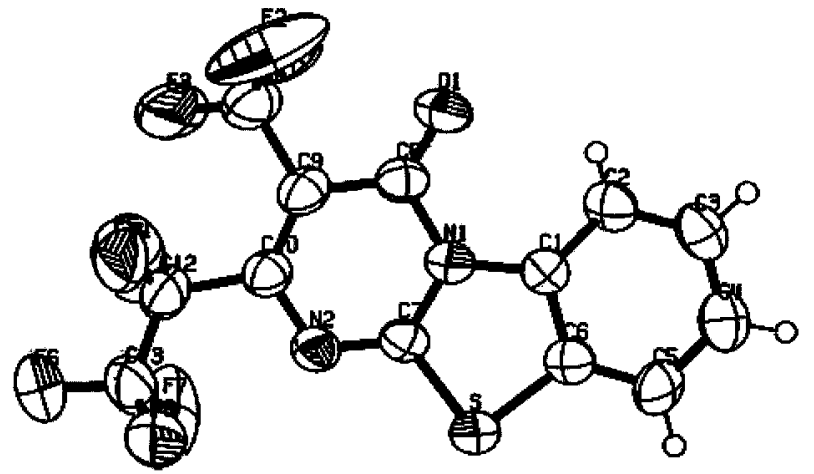

Figure 2. OR $\mid \mathrm{l}$ : $P$ dratwing ol $\mathbf{3 f}$.

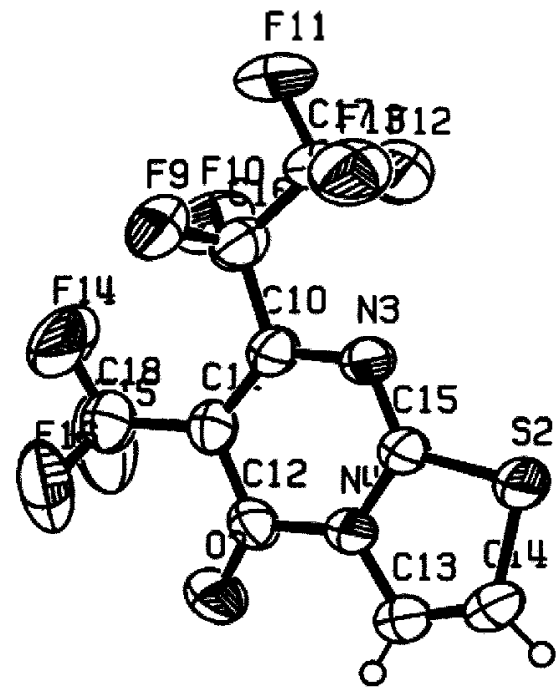

Figure 3. ORII:P drawing of 3g.

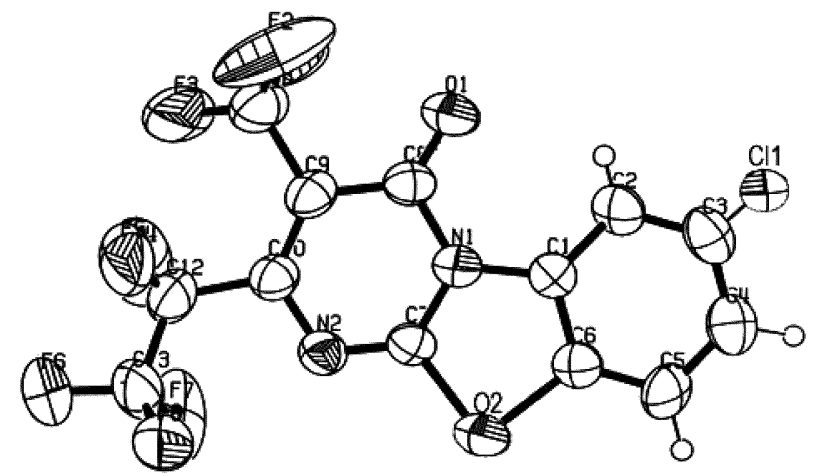

Figure 4, ORILP drawing of $3 \mathbf{h}$.

$-109.90(\mathrm{q}, J-2 \mathrm{I} .4 \mathrm{I} \mathrm{l}, 2 \mathrm{~F}),-80.23(\mathrm{~s}, 3 \mathrm{~F}),-58.11(\mathrm{t}, J-$ 21.4 Il $2,3 F)$; IR $\left(\mathrm{cm}^{-1}\right) 313 \mathrm{I}, 1686,1502,1182,1117$ : MS $m / z$ (rel. intensity) $338\left(\mathrm{M}^{-}, 100\right), 24 \mathrm{l}(100), 143(39), 100$ (21).

6-Chloro-2-pentafluoroethyl-3-trifluoromethyl-9-0xa1,4a-diazafluoren-4-one (3h). light brown solid; m.p. 60-66 "C (E1OAc/hexane); "] [-NMR (CDCl $300 \mathrm{MH} z) \delta 7.47$ (d, $J-1.0 \mathrm{l}[\mathrm{z}, 1 \mathrm{I}]), 7.62(\mathrm{~d}, J-\mathrm{I} .8 \mathrm{]}[\mathrm{z}, \mathrm{I}][$ ), $8.49(\mathrm{dd}, J-\mathrm{I} .0$.

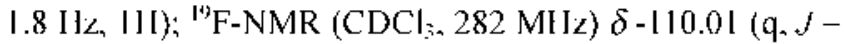

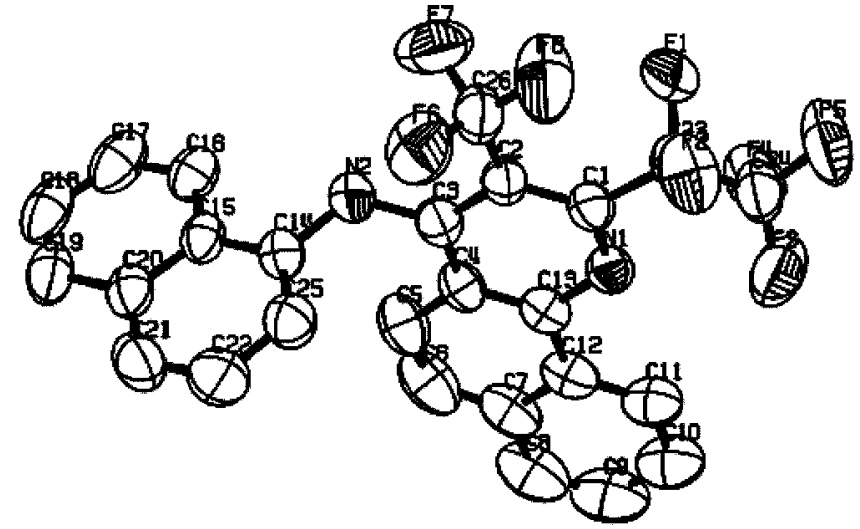

Figure 5. ORTEP drawing of $\mathbf{3 i}$. 
$22.4 \mathrm{~Hz}, 2 \mathrm{~F}$ ). -80.50 (s. 3F),-57.88 (t. $J=22.4 \mathrm{~Hz} .3 \mathrm{~F}$ ); IR $\left(\mathrm{cm}^{-1}\right)$ 3132. 1708. 1487. 1124. 911: MS $m z$ (rel. intensity) $406(\mathrm{M}, 100), 287(57), 12+(23)$.

4-(Naphthalen-1-yl)amino-2-pentafluoroethyl-3-trifluonomethylbenzo| $h]$ quinoline (3i). yellow solid: m.p. 146-148 ${ }^{\circ} \mathrm{C}$ (EtOAc/hexane); ${ }^{1} \mathrm{H}-\mathrm{NMR}\left(\mathrm{CDCl}_{3}, 300 \mathrm{MHz}\right) \delta 6.6+(\mathrm{d}$, $J=7.5 \mathrm{~Hz} . \mathrm{lH}), 6.98(\mathrm{~s}, \mathrm{lH}) .7 .2 \mathrm{l}$ (t. $J=7.9 \mathrm{~Hz} . \mathrm{lH}) .7 .48-$ $7.81(\mathrm{~m} .7 \mathrm{H}) .7 .97$ (d. $J=9.0 \mathrm{~Hz} . \mathrm{lH}) .8 .37(\mathrm{~d}, J=9.0 \mathrm{~Hz}$, lH). $9.21(\mathrm{~d}, J=9.0 \mathrm{~Hz} . \mathrm{lH}):{ }^{19} \mathrm{~F}-\mathrm{NMR}\left(\mathrm{CDCl}_{3}, 282 \mathrm{MHz}\right)$ $\delta-107.13(\mathrm{dq} . J=14.1,22.6 \mathrm{~Hz}, 2 \mathrm{~F}),-79.19(\mathrm{~d}, J=22.6 \mathrm{~Hz}$, $3 \mathrm{~F}),-54.69(\mathrm{dt}, J=2.8 .22 .6 \mathrm{~Hz} .3 \mathrm{~F}):$ IR $\left(\mathrm{cml}^{-1}\right) 3411.3068$. 1570, 1233. 1114: $\mathrm{MS} m z$ (rel. intensity) $506\left(\mathrm{M}^{\prime}, 48\right) .467$ (13). $406(35), 287$ (21), 236 (14), 127 (31), 84 (100).

4-(Naphthalen-2-yl)amino-2-pentafluoroethyl-3-trifluoromethylbenzol $f$ lquinoline (3j). brown solid: m.p. 138-139 ${ }^{\circ} \mathrm{C}$ : ${ }^{1} \mathrm{H}-\mathrm{NMR}\left(\mathrm{CDCl}_{3}, 300 \mathrm{MHz}\right) \delta 6.73(\mathrm{~s}, \mathrm{lH}) .6 .76(\mathrm{~d}, J=$ $2.2 \mathrm{~Hz} . \mathrm{lH}) .7 .187 .81$ (m. $8 \mathrm{H}) .7 .97(\mathrm{~d} . J=9.0 \mathrm{~Hz}, \mathrm{lH}) .8 .09$ (d. $J=9.0 \mathrm{~Hz} .1 \mathrm{H}) .9 .09$ (d. $J=8.8 \mathrm{~Hz} .1 \mathrm{H}$ ) ${ }^{19} \mathrm{~F}-\mathrm{NMR}$ $\left(\mathrm{CDCl}_{3} .282 \mathrm{MHz}\right) \delta-107.43(\mathrm{q} . J=23.0 \mathrm{~Hz} .2 \mathrm{~F}) .-79.38$ (s. $3 \mathrm{~F}),-54.52(\mathrm{t} . J=23.0 \mathrm{~Hz}, 3 \mathrm{~F}):\left[\mathrm{R}\left(\mathrm{cm}^{-1}\right) 3064,1354,1292\right.$. 1176; MS $m z$ (rel. intensity) $506(\mathrm{M}, 100), 467(40)$.

Acknowledgment. This work was supported by University of Ulsan Research Fund of 2001.

\section{References}

1. (a) Filler. R.: Kobavashi. Y: Yagupolskii. L. M. Organofhorine Componds in Medicinal Chemistry and Bionedical ipplica- fions. Elsevier: Amsterdan. 1993: p 386. (b) Hudlicky. M.: P's: lath. A. E. Chemistry of Onganc Flourin Componds $\mathrm{H}$. American Chemical Sociely: Washinglon. DC. 1995. (c) Smart. B. F. J. Fhorine ( 'hem 2001, 109,3

2. (a) Mann. J. ('hem Soc Rew 1987, 16, 381. (b) Welch. I. T.: Fswarakrishnan. S. Fhorihe in Biooryanic (hemistrn? Wiley: New York. 199l. (c) Banks. R. E.: Smart. B. É: latlon J. C. Organofhonine Chemistry: Honciples and Commencial Applicafions. P'lenum l'ress: New York. 1994. (d) Howe-Grant. M. Flhonine (hemistry if comprohensive Tratment: Joht Wiley: New York, $1995 ; \mathrm{p} 236$.

3. (a) Ishikawa, N.: Maruta, M: Yoshimura, N.: Kitazume. T. J. Fhom Chom. 1980. 16. 75. (b) Ishikaga. N.: Kitazume. I': Chino. K. J. Flnorme Chem. 1981. 18. 447. (c) Ikeda. I.: Umino. M.: Okahara. M.J. Ory. Chem. 1986. 5l. 569. (d) Coe. P' L.: Kay. N. C. J. Fhorine (hem 1991, 53. 15. (c) Coc. P. I... Ray, N. C.J. Fluorine (hen 1998. 88. 169.

4. Chambers, R. D.: Vaughan. I. F. S. In Organofluomine ('hemistry Fluonmated Alkenes and Reactive Intemediates. Sprincer-Verlag: Berlin. 1997: pp 2-36.

5. (a) Chi. K.-W.: Kim. S.-J.: Park. T.-H.: Gatilon: Y. V.: Bagryanskaya, I. Y: Futin. G. G. J. Fluorine (hem. 1999. 99. 29. (b) Chi. K.-W.: Futit. G. G.: Bagreanskay, I. Y.: Gatilox: Y. V.: 7.huzhof: F. I.. J. Flowhe (hem 2000. 103. 105. (c) Chi, K. -W.: Furin, C. G.: Bagryanskay. I. Y.: Galilow. Y, V. J. Fhorme ( $/$ kem. 2000. 104. 263. (d) Chi. K.-W.: Kim. H. A.: Furin. G. G.: Zhuzhgon. E'. L.: l'rotzuk. N. J, Fhonine Chem. 2001. Ho. 11. (e) Furin. G. G.: Chi, K.-W. Synthetic Wethods for Fluoroorganic (romponands. University of Ulsan Press: Ulsant. Korea, 2001.

6. Two and five equivalents of $\mathbf{2} \mathbf{i}-\mathbf{j}$ and tricthymine were respectively used lor the synthesis of $\mathbf{3 i}-\mathbf{j}$.

7. Additional data for X-ray analysis are available with Prolessors $\mathrm{Ki}$-Whan Chi and Uk Lee. 